\title{
A Study on Government Emergency Disposal System of Network Emergencies
}

\author{
Song Mei ${ }^{1,2, a}$, Quanliang Mei ${ }^{1}$ \\ ${ }^{1}$ School of Literature, Law and Economics, Wuhan University of Science and Technology, P. R. \\ China; \\ ${ }^{2}$ Hubei Institute of Administrator, P. R. China. \\ ameisong@126.com
}

\begin{abstract}
The formation of digital society provides an easy and efficient information channel for social management. In essence, the management of mediated society is information management based on big data. Monitoring and analysis of network public opinion centered on the information from the "we media" can effectively improve the ability of the government on social risk perception and reaction. In the face of external information environmental changes brought about by the new situation of social management, the government should establish "public-centered and data-guided "pattern of social management and innovate management mechanism by " Internet plus government ". These mechanisms include that the government must take social public opinion information management as the core, establish the government emergency disposal system of network emergencies, use big data network analysis technology to realize the effective combination of online public opinion analysis and offline social hot issues disposal, realize the comprehensive information management cooperation between media agencies, social organizations and experts and think tank. It will greatly improve the science and efficiency of the government social management under the network new media environment.
\end{abstract}

Keywords: Network emergencies; Network governance; E-government.

\section{Introduction}

In recent years, network emergencies are in high-risk situation around the world. China is currently in the age background of highlighted social contradictions and the rise of network society, various contradictions brought about by many social problems emerge in the process of modernization process are intensifying, the situation brings hotspot network incidents at high momentum. 2009 China's Internet public opinion statistics analysis report shows that the number of incidents by the network broke triggering public concern is 23 , accounts for $30 \%$ of all events [1]. According to the data of People's Daily online monitoring room, 500 social hot issues events occurring in the course of statistics show that $44.4 \%$ of the events triggering public attention by Internet disclosure in 2015 [2]. Thus, after 6 years of development, with the rising number of Internet users audience, the number of social hot issues events triggered by hot network public opinion has a upward trend.Coping with these hot network crisis events increased the difficulty of the governments social management. Network emergencies have some obvious differences with traditional emergencies. It has the characteristics such as information dominance, network mediated, complex subject, chain dynamics and uncertainty. Characteristics of network emergency information decided emergency disposal mode based on real-time information discovery and analysis.Network emergency management need to build emergency management system based on information driven.

\section{Major Principles of Emergency Disposal of Network Emergencies}

\subsection{Information-Dominated Principle}

With the approaching of big data era, the overall digitization of social resources will lead to the homogeneity of various social forms, which will contribute to the formation of network society in the true sense. The ease of publishing content on social media sites brings to the Web an ever increasing amount of content captured during-and associated with-real-world events [3]. The essence of 
mediated social management is the processing of big data, the UGC pattern of user generated content of internet information determines the dominant position of public "we media" in mediated society, ubiquitous "we media" function as the monitor and sensor of hot issues of mediated society as well as the amplifier and silencer of the influences of those. How the public opinion information concerned the issues is dealt with will directly affect the damage degree of issue-triggered social risks. The network of emergency disposal must analyze the evolution law of event information and network public opinion information which arised in each stage of germination, development, climax and retreat of the event. The interaction of these two kinds of information should be studied to establish the emergency disposal pattern of information driven decision.

\subsection{Dynamic Response Principle}

The chain dynamic nature and the uncertainty of network emergencies require the government to establish the real-time response mechanism of emergency decision and disposal system. Dynamic nature consists of real-time information analysis, flexible decision scheme and timely offline disposal. Making prompt study and decision through real-time acquisition and analysis of issue information and its public opinion information, meanwhile, adjusting the emergency plan and implementing the most timely offline disposal based on the experience of similar historical issues as well as the suggestion from experts and think tanks.

\subsection{Linkage Disposal Principle}

The key to the emergency disposal of network emergencies lies in source treatment and offline disposal. The main issue sources of network emergencies are hot online public opinion information and current hot issues. Hot network public opinion information is from the one who starts the online hot topics while current hot issues are from the people concerned. To establish the department linkage mechanism combining online information monitoring with offline issues disposal, which can contact with the top initiator or the issue concerned people and make offline disposal immediately. In effect, all measures above are to decrease the popularity of sources of the issue.

\subsection{Public Opinions guidance Principle}

The biggest crisis of network emergencies does not lie in short term negative effects but in the damage to the public trust of government which resulted from the inner management problems like delayed response, non-transparent information, inappropriate disposal and insincerity. Therefore, the emergency disposal of network emergencies tests the capabilities of public relations ability of handling crisis and image repairing ability of the government. Government is supposed to analyze public opinions and to guide it in a positive way.

\section{General Framework of Emergency Disposal System of Network Emergencies}

With the rise of the network society in China, the network public opinion is becoming more and more important. Internet is not only a platform of exchanging and expressing ideas, but aspecial subject with ability to set media agenda $[4,5]$. The special subject can determine the final direction of the incident by influencing the public opinion field [6].

Therefore, the information of network public opinion is becoming the key factor that currently determines the success or failure of network emergencies crisis management. As early as in 1996, the relevant institutions began to study Topic Detection and Tracking (TDT). The TDT study is intended to explore techniques for detecting the appearance of new topics and for tracking the reappearance and evolution of them [7]. For the first time, the TDT study proposed to the event information capturing and tracking analysis method. New event detection requires identifying those news stories that discuss an event that has not already been reported in earlier stories [8]. TDT technology research provides an important lesson for network emergency monitoring analysis.

The emergency disposal system of network emergencies is supposed to cover all phases of the issue life circle which can present the process of the government emergency disposal. The system should be in compliance with the above-mentioned four main principles, highlight the "information driven decision" principles of mediated society governance to form the governance organization system that strengthening the government internal linkage, promoting collaboration between the 
government and media organizations, third party consultants and other social organizations. The network of emergency disposal system should realize the comprehensive analysis based on issues and public opinions and establish the emergency management pattern combining online guidance and offline issue disposal at the same time.

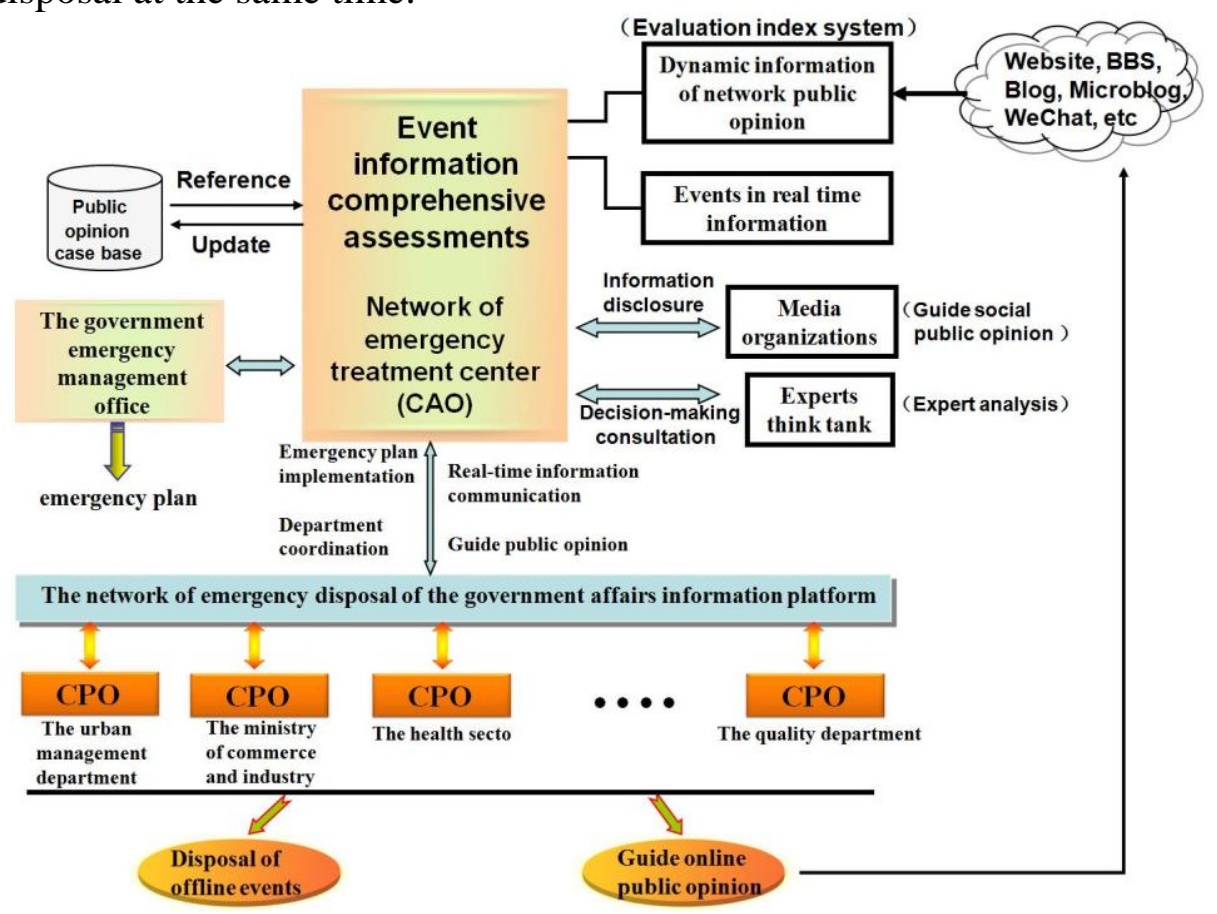

Figure 1 General Framework of the Emergency Disposal System of Network Emergencies

In terms of organizational structure, as seen above, cyberspace administration office (CAO) plays the core role and all related parties respond coordinately within the emergency management system. In the linkage among functional departments, to establish the department-coordinated emergency disposal system combining unified command, rapid response and real-time and coordinated disposal. All functional departments should set up cyberspace propaganda office (CPO) to receive information of hot issues from cyberspace administration office and to inform department leaders of the issue-related information so that these departments can guide network public opinion propaganda and dispose network emergencies in time. Confronted with major emergencies, cyberspace administration office will directly inform the emergency management office of the issue-related information who will coordinate all functional departments to launch the emergency plan for public emergencies, and cyberspace administration office will offer government the reference on the disposal of major emergencies by means of assisting emergency management office, analyzing network public opinion information and guiding network public opinion and upgrading the information on the development trend of issues and public opinions.

In terms of workflow, arranging the work of all phases based on the evolution process of the network emergencies. In the initial phase, cyberspace administration office is in charge of the monitoring of network hot issues information of jurisdictions (provincial or municipal level) and retweets hot topics information and issues development trend information to the cyberspace propaganda office of related department. After receiving the information from cyberspace administration office, related functional departments will investigate specific circumstances of the issue and take immediate measures as well as feeding back the issues information to cyberspace administration office. With regards to the nature and the development trend of issues, cyberspace administration office will work on releasing the issue-related information through media agencies as well as assisting media agencies in guiding public opinions in a positive way through traditional and network media. In the middle of the evolution process of the issue, cyberspace administration office will upgrade and analyze the information on the issue and its information on the development trend and public opinions, meanwhile, take advantage of the suggestions and opinions from experts and think tanks to adjust the emergency disposal plan. According to the plan, cyberspace administration 
office will guide and coordinate the concerned department to implement the plan. Departments concerned should dispose offline issues coordinately; meantime, cyberspace administration office ought to guide media agencies and involved functional departments in upgrading the information on emergencies disposal and respond to network public opinions. After the issue goes away, cyberspace administration office should help related departments to evaluate the work of emergency disposal and draw lessons from it as well as putting it on record to offer reference to the similar issues in the days to come. On the other hand, the related departments have to put forward specific methods of rectifying and reforming, and supervise the involved departments to rectify and reform based o the problems reflected. Cyberspace administration office will release the results of rectifying and reforming to the public to maintain the image of the government.

The emergency disposal system of network emergencies is supposed to cover all phases of the issue life circle. Keeping informed of the real situation and the network public opinion, carrying on comprehensive analysis of them, fully taking advantage of the suggestions and advice from experts and giving full play to the function of related departments in coordinating and feedback effect. To adjust emergency plan in accordance with real-time information analysis and to improve the time-sensitivity of government's work in dealing with network emergencies via the real-time issue response mechanism based on the emergency plan.

\section{Operation Mechanism of Emergency Disposal System of Network Emergencies}

After strengthening the macro-level emergency disposal system concerning institutional framework, functions and responsibilities as well as management system, the urgency is to formulate the micro-level operation system which is in accord with the governing law of mediated society and can promote the science of governing mediated society and the course of legislation. Based on the emergency disposal workflow of "plan, monitoring, analysis, disposal and evaluation" of network emergencies, the mechanism construction requires urgent enhancement in the following four aspects:

\subsection{Build Standardized Information Monitoring Mechanism}

Firstly, to establish uniform information monitoring standards. Due to the disparity among regions of the country, the difference of issue kinds and different monitoring stresses, it's essential to establish a full set of standards included in the illustrative document so that cyberspace administration offices from all levels can follow the scientific set of standards. Meantime, with the changes of the internet terms and hot keywords, the specific standards of information monitoring should be upgraded and completed in time. Secondly, to employ same monitoring-analysis software system. Various monitoring systems and their functions go against the communication among various levels of cyberspace administration offices in Hubei province and can't contribute to the improvement and upgrade of software system functions, which will directly affect the effect of disposal. Therefore, the monitoring and analysis software system of network emergencies of Hubei province should be unified through procurement bidding. Based on the uniform software system, the big data center of Hubei province can be established and the municipality-level and county-level cyberspace administration offices have access to the software platform. It can solve the problem of lacking manpower and material resources and strengthen the communication among cyberspace administration offices of all levels, promote the harmony of monitoring work and find out the problems existing in monitoring work and the solutions to those through centralized management. Finally, to set specific requirements of monitoring work. To specify the content of the information monitoring work of network emergencies, such as the requirements of monitoring time, monitoring content and reporting format so that the cyberspace administration offices of all levels can meet the same requirements.

\subsection{Build Scientific Information Analysis Mechanism}

The science of information analysis lies in the following three aspects: first, to establish the evaluation index system. Evaluation index system means that network emergencies disposal should base on the quantitative analysis and feature extraction of network public opinions of historical network emergencies, and choose the critical elements which can exactly monitor, evaluate and 
predict the development trend of network emergencies as well as setting the systematic index set which can study and tell the evolution law and development trend of public opinions of network emergencies through analyzing the relations among all related factors. Second, to scientize the tool of information analysis. Taking advantage of information science and statistics to set the theoretical analysis model of issue information as well as using big data information system to analyze the network emergencies information through statistical analysis. At last, to generalize the data information. Data information analysis of network emergencies should study and comprehensively analyze and do contrast analysis of the information of development trend and the information of network public opinions, and analyze the interaction between issue itself and the evolvement of public opinions, thus, the coordinated management combines online guidance of public opinions and offline disposal of the issue can be reached.

\subsection{Build Standardized Information Submitting System}

To set standardized information submitting system of network emergencies in accordance with the regulations and requirements of the State Council of information submission as well as the actual situation of Hubei province. According to the emergency disposal system of network emergencies, information submitting system consists of two parts: online public opinion information submission and offline issue information submission. As the main body of submitting information, cyberspace administration offices of all levels should set the reference standards: first, the grading standards are supposed to set based on the evaluation index system and the degree of importance of issue information, also, different submitting flows and time limits should be applied to different levels of cyberspace administration offices. Besides, to set information submission list concerning related functional departments in accordance with the categories and nature of network emergencies, which makes it clear for cyberspace administration offices whom to submit to. Being the main body of offline information submission, all related functional departments should refer to the Law on Response to Emergencies for the standards of submitting information of network emergencies and inform cyberspace administration offices of the development trend of current issues. In terms of submitting work, first, the specific responsibilities of the one who submits should be unequivocal and the professional qualities should be strengthened to ensure the effectiveness of information submission, besides, the submitting template should be fixed which can standardize the content and data format of the information so that the integrity and standardization can be guaranteed, after that the submitting flow and time limit should be clear. Due to the issue information grading system and different related functional departments, various submitting flows should be applied, finally, the evaluation system of information submission should be established to ensure the effectiveness and accuracy of submitting work.

\subsection{Build Collaboration Information Disposal Mechanism}

Emergency disposal system of network emergencies is mainly about information communication and coordination. Three mechanisms should be carried through. First, to carry through submission-supervision mechanism. After monitoring the information of hot issues, cyberspace administration offices should keep related functional departments informed as well as offering primary disposal suggestions based on historical disposal experience and opinions from experts so that related functional departments can refer to. After surveying the issue, related functional departments should feed back the development trend of the issue to cyberspace administration office so that cyberspace administration office can know the offline issue information based on which cyberspace administration office can adjust the disposal plan through working on the release of information to the public, analyzing the development trend of the issue as well as studying the public opinions in order to improve the timeliness and initiative when the government deals with network emergencies. Besides, to carry through release-communication mechanism. Apart from informing higher ranking leaders and related functional departments of the information of hot issues, cyberspace administration office should release the information concerning the issue through official government media agencies to guide public opinions in a positive way. At the same time, cyberspace administration office should communicate with media agencies about the issue and how it is disposed to take advantage of traditional media and emerging media to sway public opinions in a positive way 
and win the support from the media. Finally, to carry through joint supervision mechanism. According to how influential and significant and how long it lasts to grade public opinions. For major network emergencies, the online public opinions information and the offline background information of the issue should be considered together and the joint meeting of disposal of related functional departments should be convened to discuss the emergencies and arrange related work.

\section{Conclusion}

With the approaching of big data era, the overall digitization of social resources will lead to the homogeneity of various social forms, which will contribute to the formation of network society in the true sense. Digitalization of information provides easy and efficient information channel for the government management, and the essence of mediated social management is the processing of big data. Monitoring analysis of "we media"- centered network public opinion information can effectively improve government's ability of social risk perception and reaction. In the face of external information environmental changes brought about by the new situation of social management, the government should establish "public-centered and data-guided "pattern of social management and innovate management mechanism by " Internet plus government ". These mechanisms include that the government must take social public opinion information management as the core, establish the government emergency disposal system of network emergencies, use big data network analysis technology to realize the effective combination of online public opinion analysis and offline social hot issues disposal, and realize the comprehensive information management cooperation between media agencies, social organizations and experts and think tank. It will greatly improve the science and efficiency of the government social management under the network new media environment.

\section{Acknowledgments}

This work was financially supported by "the National Social Science Foundation of China (13CGL132)", "the Fundamental Research Funds of National School of Administration (13HZKT199)", “and the Scientific Projects of Hubei Province (2015BDF037)".

\section{References}

[1]. Information on http://yq.people.com.cn/htmlArt/Art392.htm

[2]. Information on http://yuqing.people.com.cn/n1/2015/1224/c401685-27972437.html

[3]. Becker, Hila, M. Naaman, and L. Gravano. "Learning similarity metrics for event identification in social media." Wsdm 10 Third Acm International Conference on Web Search \& Data Mining 2010:291-300.

[4]. James Curran, "Media and power", Tsinghua University Press, Beijing, 2006.

[5]. Ileana Rotaru, "The post-modern paradigm-a framework of today's media impact in cultural

[6]. Space", Journal of Procedia - Social and Behavioral Sciences, vol.5, no.1, pp.328-330, 2010.

[7]. Zhao Hongyan, He Mingsheng. "Formation path of network public opinion based on collaborative interaction using social network analysis", Advances in Information Sciences \& Service Sciences, 2012, 4(22):247-255.

[8]. James Allan, Jaime Carbonell, George Doddington, Jonathan Yamron, Yiming Yang. "Topic detection and tracking pilot study Final report", in Proc. DARPA Broadcast News Transcription and Understanding Workshop, 1998, pp. 194-218.

[9]. James Allan, Ron Papka, Victor Lavrenko. "On-line new event detection and tracking", An International ACM SIGIR Conference on Research and Development in Information Retrieval, 1998:37-45. 